\title{
Advances In Wireless Test beds and Research Infrastructures
}

\author{
Miguel Ponce de Leon • Shiwen Mao • Frank Steuer • \\ Jens Schumacher $\cdot$ Thomas Magedanz • \\ Raheem A. Beyah $\cdot$ Scott Midkiff
}

Published online: 25 February 2010

(C) Springer Science+Business Media, LLC 2010

Communication infrastructures play a vital role in modern society. Advances in the range of service offerings, performance, quality of service, security, and ubiquity continue to flourish, despite global economic fluctuations. Clearly the world has gone wireless with a massive set of new smart phones, service platforms, middleware, and application stores.

With this growth, fresh research challenges in the wireless environment have arisen, from the need for efficient energy conservation, to the integration of wireless systems into heterogeneous large scale networks, to the fast reauthentication of clients as they move between communication systems.

Wireless test beds and experimental research infrastructures are playing a vital role in validating new innovations in this environment. Access to experimental wireless infrastructure for verifying real-life applications by specific user communities benefits all the stakeholders involved: the end

\section{Ponce de Leon $(\square)$}

Telecommunications Software \& Systems Group (TSSG),

Waterford Institute of Technology,

Waterford, Ireland

e-mail: miguelpdl@tssg.org

\section{S. Mao}

Department of Electrical and Computer Engineering,

Auburn University,

Auburn, AL, USA

e-mail: smao@auburn.edu

F. Steuer

Technische Universität Berlin, DAI-Labor,

Berlin, Germany

e-mail: frank.steuer@dai-labor.de

\section{J. Schumacher}

University of Applied Sciences Vorarlberg,

Dornbin, Vorarlberg, Austria

e-mail: Jens.Schumacher@fhv.at users, because of the firsthand evaluation of the provided services, the researchers and infrastructure experimenters, because of the knowledge gained from hands-on study and analysis, and the service providers, because of the highlighted potential business exploitation of the network.

The purpose of this special issue is to present the landscape of recent progress in mobile and wireless test beds. In a collection of twelve articles, this issue shows advances from the computing, networking and services perspective, providing insight into the importance and challenges of mobile networking in future communication environments.

We start the issue with a review of the evolution of experimentation facilities related to the improvements in wireless mesh networking. It is clear that no single wireless network will exist on its own, but will be deployed in combination with others. In the first article by Blywis et al., it is shown how wireless mesh networking can act as an

\section{T. Magedanz}

Institute for Telecommunications Systems,

Technische Universität Berlin,

Berlin, Germany

e-mail: tm@cs.tu-berlin.de

R. A. Beyah

Communications Assurance and Performance Group,

Department of Computer Science, Georgia State University, Atlanta, GA, USA

e-mail: rbeyah@cs.gsu.edu

\section{S. Midkiff}

Bradley Department of Electrical and Computer Engineering, Virginia Polytechnic Institute and State University,

Blacksburg, VA, USA

e-mail: midkiff@vt.edu 
integrator for other wireless networks like sensor networks or personal area networks. However to extensively test this integration, the article advocates a move away from the simulation environment, and shows how more real world studies can be achieved in a dedicated test bed-based research environment.

The second article of this issue shows a complete test campaign to evaluate power consumption associated with mobile data communication. The paper by Díaz Zayas et al., provides an extensive analysis of the different factors influencing the power consumption of data connections on mobile devices taking into account the access technology in use, the applications and protocols involved, including physical link level management details and specific cellular technology aspects such as handovers. Interestingly, the paper highlights a software tool which turns commercial smart phones into powerful test systems, negating the need for custom made mobile handset prototypes in the testbed.

The third article by Botta et al., looks at how distributed test beds can play a fundamental role in the evaluation of disruptive innovation for the future Internet. The paper highlights how the authors managed to successfully provide 3G connectivity to stationary PlanetLab Europe nodes. A description of the usage model for granting access to the UMTS connection within a slice and the technical challenges faced while trying to integrate such support in a PlanetLab-based environment is detailed.

The fourth article by Blum et al., tackles the issues around enabling rapid communication service design. The paper describes work on a policy-based service broker which allows for the definition of request- and service-specific policies serving as service contracts that have been implemented as part of the Open SOA telco playground test bed.

As was highlighted in an earlier article, the fifth paper of this issue also makes it clear that current cellular telecommunication networks are made up of several radio networks integrated together to provide a complex system in terms of architecture and management. The large number of possible faults in software, hardware, the configuration and the environment, makes troubleshooting a complex task. The paper by Skehill et al., highlights how automation of troubleshooting will allow mobile operators to alleviate the burden of troubleshooting teams and to shorten the time necessary to identify faults and, thus, reduce the time in which the network suffers from poor performance. The paper goes on to describe a UMTS/WLAN test platform that is built according to 3GPP and IEEE standards. The platform is used to introduce common faults and produce key performance indicators (KPI) similar to real world networks.

The sixth article by De Moor et al., focuses on the perceived quality of experience (QoE) of mobile systems, highlighting the fragmented nature of the literature on QoE and its related concepts of quality of service and user experience in the mobile environment. This fragmentation has resulted in still largely unknown factors which affect mobile QoE and how users' subjective experiences of such applications and services could be adequately identified and optimized. The paper goes on to present the relationship between technical parameters and the human experience and sets forth a conceptual framework for the multi-dimensional evaluation of quality of experience in a mobile, real-life environment.

The seventh article in this issue highlights the need to control access of mobile users to network access services such that only authenticated mobile users are allowed to access the service. However, in this environment handover key management and re-authentication causes a major problem when it comes to bandwidth demanding applications such as VoIP and video conferencing. To this end, the paper by Marin-Lopez et al., presents an architecture for fast re-authentication based on the well-known Kerberos protocol, where multi-realm handoff scenarios are used to find a tradeoff between the extensible authentication protocol (EAP) with recursive Kerberos crossrealm ticket operation and full EAP authentication. A description of the wireless test bed prototype used to prove the feasibility of the architecture and to obtain experimental results for the bootstrapping and handoff phases is also highlighted in the paper.

In the eighth article of this special issue, Schreiner et al. depicts the architecture, principles, and functionality of a Service Oriented Architecture (SOA)-based research infrastructure as it is currently prototyped at Fraunhofer FOKUS to enable dynamic service composition for multiple network technologies. The principles developed in the context of this work are applied to composition mechanisms enabling custom tailored test beds in a distributed test bed federation and composition of adaptive networks based on temporary service requirements.

The ninth article by Alay et al., addresses the problem of video multicast over wireless local area networks (WLANs) with multi-rate capability. The authors present a scheme that dynamically adapts the transmission rate and forward error correction (FEC) rate for multicast video sessions. The paper also describes the open source device driver implementation of the proposed scheme, and presents test bed experimental results to demonstrate the achievable improvements on multicast video.

The tenth article by Walker et al., investigates scalability issues with the MeshTest wireless test bed. The test bed uses shielded enclosures and a matrix switch of programmable attenuators to produce multi-hop scenarios and simulate the effect of mobility and fading. The authors evaluate two potential designs for a scalable version of the MeshTest test bed. They show how to adopt a multi-switch system to make MeshTest scalable and how to improve test bed performance. 
The eleventh paper by Zhao at al., describes an experimental study on utility function selection and its impact on streaming video performance with an IEEE 802.22 WRAN base station (BS) cognitive engine (CE) test bed developed at Virginia Tech. They show that significant improvements on received video quality can be achieved when the $\mathrm{CE}$ adopts a dynamic, content-aware, video-specific utility function rather than a static, predefined, general purpose utility function.

The last article in this special issue by Wellens and Mähönen presents an extensive spectrum occupancy measurement study, as well as lessons learned from the measurements. Such a measurement study provides motivation for dynamic spectrum access (DSA). The authors compare measurement results gathered at three locations and show differences in the background noise processes. A new model for the duty cycle distribution is also developed that has multiple applications in DSA research.

These twelve papers comprise the special issue on advances in wireless test beds and research infrastructures, an issue which covers a breath and depth of research in the field. We conclude with a special word of thanks to all of the authors who submitted their articles, to the many reviewers for giving their time to provide invaluable feedback, and to the Springer production staff of MONET for all of their help and patience. Finally a word of thanks goes to Professor Imrich Chlamtac whose guidance and support has been very much appreciated.

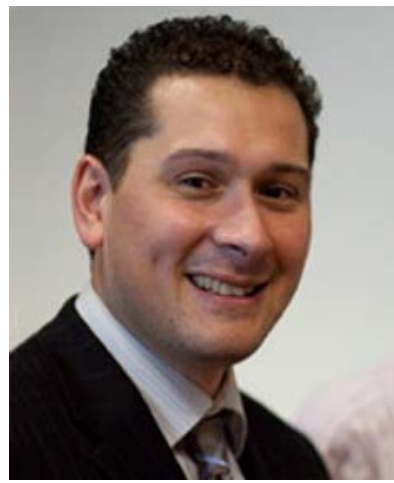

Miguel Ponce de Leon is a research manager of Communication Infrastructure Management (CIM), a unit of the Telecommunications Software \& Systems Group (TSSG), a research group which focuses on telecommunications software services management and Internet technologies. Miguel participates in a number of national and European funded research projects focusing on area of the Future Internet, where his research is concentrating on the "Architecture and Design for the Future Internet". Miguel manages a group of 50 researchers that are looking at the self-management of virtual resources that can span across heterogeneous networks, support service mobility, quality of service and reliability. Part of his research team are looking at security threats to communication-based service information such as viruses, credit card fraud, infringement of private life, economic espionage, and hacking. While his team also participates in the Living Labs concept were the ground work for more elaborate and distributed prototyping, testing, and validation facilities are being created. Miguel has worked on EU FP5 \& FP6 projects and during the EU FP7 programme his research projects include ICT 4WARD, ICT EFIPSANS, ICT AutoI, ICT PanLabs II, ICT Perimeter, ICT ThinkTrust, ICT IncoTrust and ICT CoMiFin. Miguel has served on a number of conference organising committees and in 2008 was general chair for the TridentCom conference. Miguel is a member of Engineers Ireland, FITCE and IEEE. Miguel is a Waterford Institute of
Technology graduate, with a diploma and degree in Electronic Engineering (Hon).

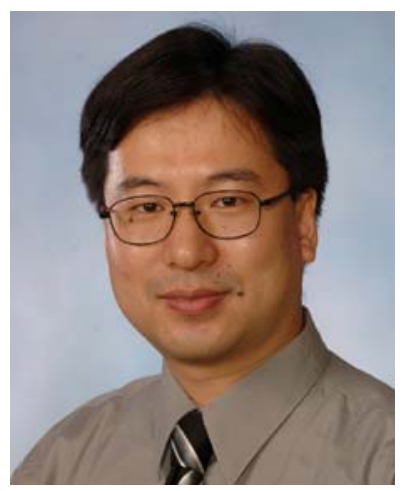

Shiwen Mao received the Ph.D. degree in Electrical and Computer Engineering from Polytechnic University, Brooklyn, NY (now Polytechnic Institute of New York University) in 2004. Currently, he is an Assistant Professor in the Department of Electrical and Computer Engineering at Auburn University, Auburn, AL. He was a Research Scientist in the Bradley Department of Electrical and Computer Engineering at Virginia Tech, Blacksburg, VA from 2003 to

2006. He was a Research Staff Member with IBM China Research Lab from 1997 to 1998.

Dr. Mao's research interests include modeling, performance analysis, optimization, and algorithms for wireless networks, with current focus on cognitive networks, free space optical networks, and multimedia communications. His research has been, and is supported by the US Department of Defense, US National Science Foundation, and TranSwitch Corporation. He is the lead-recipient of the 2004 IEEE Communications Society Leonard G. Abraham Prize in the Field of Communications Systems and the Best Paper Runner-up Award of QShine 2008, and received a Certificate of Appreciation from the IEEE Computer Society in 2007. Dr. Mao is the co-author of a textbook, TCP/IP Essentials: A Lab-Based Approach (Cambridge University Press, 2004) and the co-editor of Broadband Mobile Multimedia: Techniques and Applications (CRC Press, 2008). He is on the Editorial Board of IEEE Transactions on Wireless Communications, Elsevier Ad Hoc Networks Journal and Wiley International Journal of Communication Systems and ICST Transactions on Mobile Communications and Applications. He served as guest editor for several other journals and organizing committee member for several international conferences. Dr. Mao is a member of Tau Beta Pi, Eta Kappa $\mathrm{Nu}$, and a Senior Member of the IEEE.

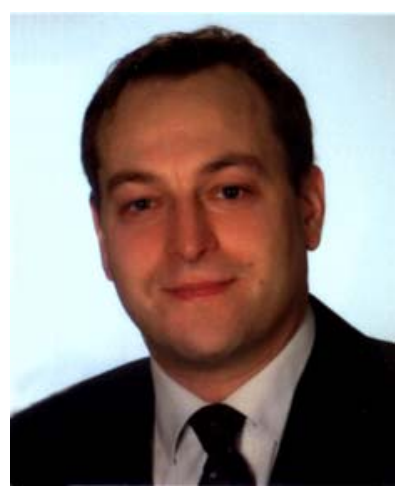

Frank Steuer serves as CTO at SemperLink $\mathrm{GmbH}$ which is a spin-off from the Distributed Artificial Intelligence Laboratory (DAI-Labor) at Technische Universität Berlin. With SemperLink he is working on mobile networks and services for the connected (electric) vehicle of the future. At the DAI-Labor he is managing various projects funded by the German Federal Government, European Union, and ICT industry. In the context of the German IT Summit he established the Beta-Plattform which is a part of the Berlin cluster in ICT PanLabs II.

Before joining SemperLink he was head of the Competence Center Network \& Mobility at the DAI-Labor. Having spent several research periods at Helsinki University, Oregon State University, and Portland State University, he received both his M.Sc. degree in communication 
and media engineering and his diploma in mechanical engineering and technical computer science from University of Applied Science Offenburg, Germany in 1997 and 2001 respectively. His research interests include new communication paradigms in future $4 \mathrm{G}$ systems, particularly mobility management in converging wireline and wireless networks, vehicular networks, context-awareness, and policy-based networking. Frank was TPC chair for the TridentCom conference in 2008. He is a member of the IEEE and the ACM.

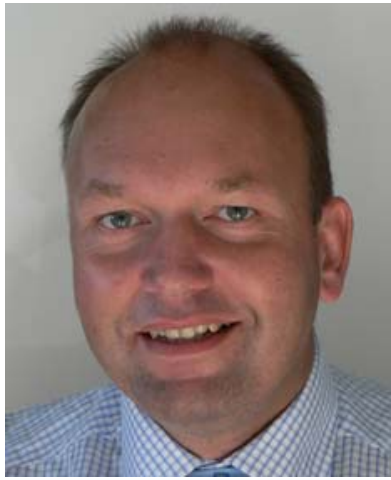

Jens Schumacher is a research professor at the University of Applied Sciences Vorarlberg in Austria since 2007. From 20032007 he was research assistant at the Bremen University and department leader for "intelligent information and communication environments for cooperative Production" at the Bremen Institute for Applied Work sciences and Production Technologies (BIBA) in Germany. He received the academic grade "DiplomInformatiker" from the faculty of Computer Sciences at the Bremen University in 1994. In 2001 he received the Academic grade "Dr.-Ing." from the faculty of Production technology at the Bremen University, Germany.

Jens Schumacher has been involved in the deployment of different test beds and mobile cities in Europe. His research work is coined by the interest to develop innovative application for mobile technological test beds. Recently he has worked on user-centered Innovation in test beds as part of different Living Lab activities. The EU-projects that he has been involved in are part of the Future Internet Research \& Experimentation (FIRE) initiative of the European Commission.

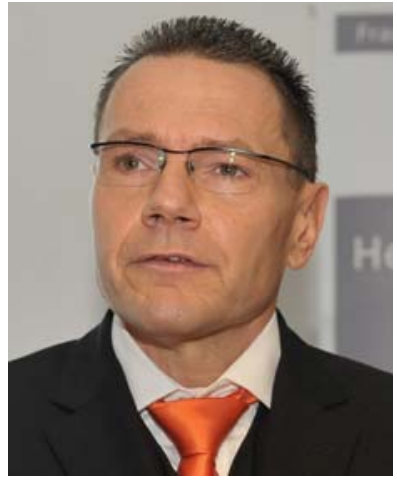

Thomas Magedanz $(\mathrm{PhD})$ is professor in the electrical engineering and computer sciences faculty at the Technische Universität Berlin, Germany. In addition, he is director of the "next generation network infrastructure" division of the Fraunhofer Institute FOKUS, which provides various testbeds and tool kits enabling advanced research and education in the context of converging networks and open service delivery platforms. Since more than 20 years Prof. Magedanz is working in the convergence field of fixed and mobile telecommunications, the internet and information technologies. In addition, Prof Magedanz is senior member of the IEEE, and editorial board member of several international journals. In 2007, Prof. Magedanz joined the European FIRE (Future Internet Research and Experimental Facilities) Expert Group.

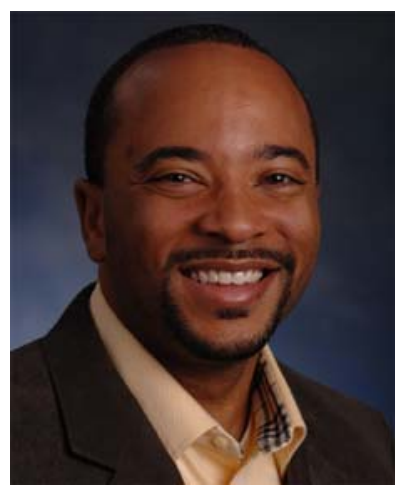

Raheem Beyah is an Assistant Professor in the Department of Computer Science at Georgia State University where he leads the Georgia State Communications Assurance and Performance Group (CAP). He is also an Adjunct Professor in the School of Electrical and Computer Engineering at the Georgia Institute of Technology. He received his B.S. in Electrical Engineering from North Carolina A\&T State University in 1998. He received his M.S. and Ph.D. in Electrical and Computer Engineering from the Georgia Institute of Technology in 1999 and 2003, respectively. Prior to joining Georgia State in 2005, Dr. Beyah was a research faculty member with the Georgia Institute of Technology's Communications Systems Center (CSC) for four years and remains a part of the Center. In 2009 he served as a Guest Editor for MONET. He is an Associate Editor of several journals including the (Wiley) Wireless Communications and Mobile Computing Journal. He regularly serves on program committees for leading conferences and on proposal review panels for several government agencies. He recently edited the book "Security in Ad Hoc and Sensor Networks." Dr. Beyah received the National Science Foundation CAREER award in 2009. He is a member of ACM, NSBE, and a senior member of IEEE.

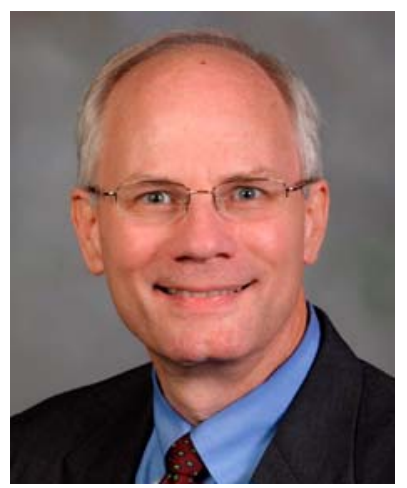

Scott F. Midkiff is the Department Head and a Professor in the Electrical and Computer Engineering Department at Virginia Polytechnic Institute and State University (Virginia Tech). He has been on the faculty at Virginia Tech since 1986 and became Department Head in August 2009. From September 2006 until September 2009, Prof. Midkiff was on assignment as a Program Director at the National Science Foundation (NSF). He has prior industrial experience at Bell Laboratories (1979-1982) and, as a student, at IBM (Summers 1977 and 1978). He was a visiting research associate at Carnegie Mellon University (1985-1986). Prof. Midkiff received the B.S.E. degree, summa cum laude, in Electrical Engineering and Computer Science from Duke University (1979), the M.S. in Electrical Engineering from Stanford University (1980), and the Ph. D. in Electrical Engineering from Duke University (1985). Prof. Midkiff has developed and taught undergraduate and graduate courses in network protocols and architectures, wireless networks and mobile systems, network applications, telecommunications, and other areas of electrical and computer engineering and computer science. He conducts research in wireless networks, mobile systems, pervasive computing, and network simulation. Prof. Midkiff is a Senior Member of the IEEE and a member of the ACM and ASEE. 